\title{
HOROSPHERES AND CONVEX BODIES IN HYPERBOLIC SPACE
}

\author{
L. A. SANTALó
}

1. Introduction. In extending certain topics from euclidean to hyperbolic geometry one finds that in certain cases the euclidean planes transfer into hyperbolic planes (geodesic surfaces), whilst in other cases the natural analogue for hyperbolic space of the euclidean planes are the horospheres (limit spheres). For instance, in the work of Gelfand and Graev on the application of the integral geometry to group representations [5], in passing from euclidean to hyperbolic space the natural analogue of euclidean planes are the horospheres. In the present note we show that the same happens with certain integral formulae on convex bodies. If $K$ is a convex body in euclidean 3 -dimensional space, it is well known that the measure of all planes meeting $K$ is equal to the integral of mean curvature $M$ of the boundary of $K$ (assumed of class $C^{2}$ ) (see, for instance, Kendall-Moran $[6$, p. 80]). In hyperbolic space the same measure is equal to $M-V$, where $V$ is the volume of $K$ [7]. However if instead of planes we consider the set of horospheres which intersect $K$, we shall prove that the measure is again $M$. We also prove, in passing, the formulae (3.4) and (4.4) referring to horospheres which intersect a fixed curve or a fixed surface of hyperbolic space.

2. Horospheres in hyperbolic space. We shall first review a few notions on surfaces in hyperbolic space which will be useful for our purposes.

In a system $\rho, \theta, \phi$ of geodesic polar coordinates the arc element has the form (Cartan [2, p. 240]),

$$
d s^{2}=d \rho^{2}+\sinh ^{2} \rho\left(d \theta^{2}+\sin ^{2} \theta d \phi^{2}\right)
$$

and the volume element is

$$
d V=\sinh ^{2} \rho d \rho \wedge d \omega
$$

where $d \omega=\sin \theta d \theta \wedge d \phi$ represents the element of solid angle corresponding to the direction $\theta, \phi$.

Between the principal radii of normal curvature $r_{i}(i=1,2)$ at a point $P$ of a surface $\Sigma$ and the distances $R_{i}$ from $P$ to the contact point of the normal to $\Sigma$ at $P$ with the envelope of the normals to $\Sigma$ along the corresponding line of curvature, the relations

Received by the editors October 31, 1966. 


$$
r_{i}=\tanh R_{i}
$$

hold (Eisenhart $[4$, p. 214]).

The integral of the mean curvature $M$ of a closed surface $\Sigma$ (of class $C^{2}$ ) is defined by

$$
M=\frac{1}{2} \int_{\Sigma}\left(\frac{1}{r_{1}}+\frac{1}{r_{2}}\right) d \sigma
$$

where $d \sigma$ denotes the area element of $\Sigma$.

If $k_{a}$ denotes the absolute or intrinsic (Gaussian) curvature and $k_{r}=1 / r_{1} r_{2}$ the relative curvature of $\Sigma$ at $P$, since the curvature of the hyperbolic space is -1 , we have (Cartan $[2$, p. 194])

$$
k_{a}=k_{r}-1 \text {. }
$$

For the planes (geodesic surfaces) of the hyperbolic space, we have $k_{r}=0$ and therefore $k_{a}=-1$. The horospheres (limit spheres) result from spheres $\left(R_{i}=\right.$ constant), when $R_{i} \rightarrow \infty$; therefore, for horospheres we have $r_{1}=r_{2}=1$ and $k_{r}=1, k_{a}=0$.

Let us consider a surface $\Sigma$ and a horosphere $H$ which intersect in the curve $\Gamma$. Let $P$ be a point of $\Gamma$ and $N_{\Sigma}, N_{H}$ the normals to $\Sigma, H$ at $P$, and $N$ the principal normal of $\Gamma$ at $P$. Let $\alpha$ be the angle between $N_{\Sigma}$ and $N, \alpha_{1}$ the angle between $N_{H}$ and $N$ and call $\theta=\alpha+\alpha_{1}=$ angle between $N_{\Sigma}$ and $N_{H}$. Meusnier's theorem gives

$$
\rho=r \cos \alpha=r^{\prime} \cos \alpha_{1}
$$

where $\rho$ denotes the radius of curvature of $\Gamma$ at $P$ and $r, r^{\prime}$ are respectively the radii of normal curvature of $\Sigma, H$ at $P$ corresponding to the direction tangent to $\Gamma$ (Eisenhart [4, p. 152], Cartan [2, p. 224]).

On the other hand, if $\rho_{g}^{\Sigma}$ and $\rho_{o}^{H}$ denote the radii of geodesic curvature of $\Gamma$ considered respectively as a curve of $\Sigma$ and a curve of $H$, we have (Eisenhart [4, p. 152], Cartan [2, p. 225])

$$
\rho=\rho_{0}^{\Sigma} \sin \alpha=\rho_{g}^{H} \sin \alpha_{1} .
$$

Since $H$ is a horosphere, we have $r^{\prime}=1$ and from (2.6) and (2.7) we deduce

$$
\stackrel{H}{\rho_{g}}=(r \sin \theta) /(1-r \cos \theta) .
$$

3. Density for horospheres. Let $S$ be a sphere of center $C$ and radius $R$ in hyperbolic space. Assume that the origin of coordinates 0 is exterior to $S$ and let $\rho+R, \theta, \phi$ be the geodesic polar coordinates of $C, \rho$ being the distance from 0 to $S$. In order to have a measure for 
sets of spheres of radius $R$ which are invariant under the group of hyperbolic motions, we may take any density of the form $f(R) d V$, where $f(R)$ is an arbitrary function of $R$ and $d V=\sinh ^{2}(\rho+R) d \rho \wedge d \omega$ is the volume element corresponding to the center $C$. When $R \rightarrow \infty$, the spheres $S$ pass to horospheres $H$ and in order to obtain a not constant density for sets of horospheres, we take $f(R)$ such that $f(R) e^{2 R} \rightarrow$ constant $\neq \neq 0, \infty$. Then, the density for horospheres results, up to a constant factor,

$$
d H_{+}=e^{2 \rho} d \rho \wedge d \omega
$$

where $\rho$ denotes the distance from the origin 0 to the horosphere $H$.

The way we have obtained this density shows that, up to a constant factor, it is the unique one which is invariant with respect to the group of hyperbolic motions of the space. The notation $H_{+}$indicates that the horosphere $H$ has its convexity towards the origin of coordinates 0 .

Analogously, if we start from spheres which contain 0 in its interior, we will have $d V=\sinh ^{2}(R-\rho) d \rho \wedge d \omega$, so proceeding as before we get

$$
d H_{-}=e^{-2 \rho} d \rho \wedge d \omega
$$

which applies to horospheres with the concavity towards 0 . We shall write $d H$ for the density of horospheres, with the convention of taking $d H_{+}$or $d H_{-}$according as the convexity of $H$ is turned towards 0 or in the opposite sense.

As an application, consider a fixed curve $\Gamma$ of length $L$ and a moving sphere of constant radius $R$. If $\nu$ is the number of intersection points between $\Gamma$ and $S$, the integral formula

$$
\int \nu d V=2 \pi L \sinh ^{2} R
$$

is known where $d V$ is the volume element corresponding to the center $C$ of $S$ and the integral is extended over all positions of $S$ for which $\nu \neq 0$. Formula (3.3) is well known in the euclidean case (in which $\sinh R$ must be replaced by $R$ ) [8] and it holds with a similar proof in hyperbolic space taking into account that the surface area of the sphere of radius $R$ is $4 \pi \sinh ^{2} R$ in this case. Multiplying (3.3) by $4 e^{-2 R}$ and making $R \rightarrow \infty$, we get

$$
\int \nu d H=2 \pi L
$$

where $\nu$ means now the number of intersection points of $\Gamma$ with the 
horosphere $H$ and the integral may be considered as extended over all horospheres, $\nu$ being zero for the horospheres which do not intersect $\Gamma$.

4. A differential formula on densities. We need a formula on densities analogous to a known formula of the integral geometry of euclidean space.

Let $\Sigma_{0}, \Sigma_{1}$ be two surfaces (of class $C^{2}$ ) in euclidean space, with $\Sigma_{0}$ fixed and $\Sigma_{1}$ moving with the kinematic density $d \Sigma_{1}$, which intersect in a curve $\Gamma$. We denote by $\theta$ the angle between the normals to $\Sigma_{0}$, $\Sigma_{1}$ and by $d s$ the arc element of $\Gamma$ at a point $P$. Then Blaschke proved the following formula [1],

$$
d s \wedge d \Sigma_{1}=\sin ^{2} \theta d \theta \wedge d \sigma_{0} \wedge d \phi_{0} \wedge d \sigma_{1} \wedge d \phi_{1}
$$

where $d \sigma_{0}, d \sigma_{1}$ denote the area elements of $\Sigma_{0}, \Sigma_{1}$ at $P$ and $\phi_{0}, \phi_{1}$ denote rotations about the respective normals to $\Sigma_{0}, \Sigma_{1}$ at $P$. This formula (4.1) has been generalized to $n$-dimensional euclidean space by S. S. Chern [3], to elliptic space by Ta-Jen $\mathrm{Wu}$ [9] and it holds with analogous proof in hyperbolic space [7].

Let us consider the case in which $\Sigma_{1}$ is a sphere of radius $R$ and center $C$. Then $d \Sigma_{1}=d V \wedge d \omega \wedge d \tau$, where $d V$ is the element of volume at $C, d \omega$ is the element of solid angle corresponding to the direction $C P$ and $d \tau$ is the rotation element about the line $C P$. We have also $d \sigma_{1}=\sinh ^{2} R d \omega, d \tau=d \phi_{1}$ and therefore (4.1) gives

$$
d s \wedge d V=\sin ^{2} \theta \sinh ^{2} R d \theta \wedge d \sigma_{0} \wedge d \phi_{0} .
$$

We recall that, as usual in integral geometry, we always consider the densities in absolute value, so there is no question of sign. Multiplying both sides of (4.2) by $4 e^{-2 R}$ and making $R \rightarrow \infty$, we get (according to $\$ 3$ ),

$$
d s \wedge d H=\sin ^{2} \theta d \theta \wedge d \sigma_{0} \wedge d \phi_{0}
$$

which is the formula on densities we want to obtain.

We get a first application of (4.3) by integrating over all positions of $H$ in which it intersects a fixed surface $\Sigma_{0}$ of area $F_{0}(0 \leqq \theta \leqq 2 \pi$, $\left.0 \leqq \phi_{0} \leqq \pi\right)$. We get

$$
\int_{H \cap \Sigma_{0} \neq 0} \lambda d H=\pi^{2} F_{0}
$$

where $\lambda$ is the length of the intersection curve $\Gamma$ of $\Sigma_{0}$ and $H$.

5. Horospheres which intersect a convex body. Let us apply the formula (4.3) to the case of a closed surface $\Sigma_{0}$ of class $C^{2}$ intersected 
by a horosphere $H$. Let $\rho_{g}^{H}$ be the radius of geodesic curvature of the intersection curve $\Gamma$, considered as a curve of the horosphere $H$. According to (2.8) and the Euler theorem which gives the normal curvature $1 / r$ of $\Sigma_{0}$ at $P$ in terms of the principal normal curvatures $1 / r_{1}$, $1 / r_{2}$ (a theorem which holds in hyperbolic space with the same form as in euclidean space, Eisenhart [4, p. 154]), we have

$$
\stackrel{{ }_{\kappa_{0}}^{H}}{{ }^{\prime}}=1 / \rho_{0}^{H}=(1 / \sin \theta)\left(\left(\cos ^{2} \phi_{0}\right) / r_{1}+\left(\sin ^{2} \phi_{0}\right) / r_{2}\right)-\cot \theta .
$$

Multiplying both sides of (4.3) by $\kappa_{g}^{H}$ and integrating over all positions of $H$ with $H \cap \Sigma_{0} \neq 0$, we get:

(a) At the left side we have

$$
\int_{H \cap \Sigma_{0} \neq 0}\left(\int_{\Gamma}^{K_{0}^{H}} d s\right) d H=2 \pi \int_{H \cap \Sigma_{0} \neq 0} n d H
$$

where $n$ is the Euler characteristic of the 2-dimensional domain on $H$ which is interior to $\Sigma_{0}$ and whose boundary is $\Gamma$. We have applied $\int_{\Gamma} \kappa_{g}^{H} d s=2 \pi n$, which follows from the fact that the absolute curvature of $H$ is 0 and therefore its intrinsic geometry coincides with that of the euclidean plane.

(b) At the right side we have

$$
\begin{aligned}
& \int_{0}^{2 \pi}|\sin \theta| d \theta \int_{\Sigma_{0}} d \sigma_{0} \int_{0}^{\pi}\left(\frac{\cos ^{2} \phi_{0}}{r_{1}}+\frac{\sin ^{2} \phi_{0}}{r_{2}}\right) d \phi_{0} \\
& \quad-\pi F_{0} \int_{0}^{2 \pi}|\sin \theta| \cos \theta d \theta=4 \pi M_{0} .
\end{aligned}
$$

Consequently, we have

$$
\int_{H \cap \Sigma_{0} \neq 0} n d H=2 M_{0} .
$$

If we call $h$-convex the surfaces which are closed and $n=1$ for any horosphere (i.e. the intersection curve $\Gamma=H \cap \Sigma_{0}$ is simply connected for any $H$ ), we have the

THEOREM. The measure of the horospheres which intersect an h-convex surface $\Sigma_{0}$ is equal to $2 M_{0}$, where $M_{0}$ is the integral of mean curvature of $\Sigma_{0}$.

Note that every $h$-convex surface is convex; the converse is clearly not true. 


\section{BIBLIOGRAPHY}

1. W. Blaschke, Integralgeometrie 17. Ueber Kinematick, Bull. Soc. Math. Grèce 17 (1936), 1-12.

2. E. Cartan, Leçons sur la géométrie des espaces de Riemann, Gauthier-Villars, Paris, 1946.

3. S. S. Chern, On the kinematic formula in the euclidean space of $n$ dimensions, Amer. J. Math. 74 (1952), 227-236. 1949.

4. L. Eisenhart, Riemannian geometry, Princeton Univ. Press, Princeton, N. J.,

5. I. M. Gelfand and M. I. Graev, An application of the horosphere method to the spectral analysis of functions in real and imaginary Lobatchewsky spaces, Trudy Moskov. Mat. Obšč. 11 (1962), 243-308.

6. M. G. Kendall and P. A. P. Moran, Geometrical probability, Hafner, New York, 1963.

7. L. A. Santal6, Geometria integral en los espacios tridimensionales de curvatura constante, Math. Notae 9 (1949), 1-28.

8. - A theorem and an inequality referring to rectifiable curves, Amer. J. Math. 63 (1941), 635-644.

9. Ta-Jen Wu, Ueber elliptische Geometrie, Math. Z. 43 (1938), 495-521.

Facultad de Ciencias Exactas y Naturales, Universidad de Buenos Aires 